\title{
LAOS behavior of the two main gluten fractions: Gliadin and glutenin
}

\author{
Gamze Yazar ${ }^{\text {a, b }}$, Ozlem C. Duvarci ${ }^{\text {a, c }}$, Sebnem Tavman ${ }^{\text {b }}$, Jozef L. Kokini ${ }^{\text {a, * }}$ \\ a Purdue University Food Science Department, 745 Agriculture Mall Dr West Lafayette, IN 47907 \\ ${ }^{\mathrm{b}}$ Ege University Food Engineering Department, Ege University Campus, Bornova, Izmir, Turkey \\ ${ }^{\mathrm{c}}$ Izmir Institute of Technology, Department of Chemical Engineering, Urla, Izmir, Turkey
}

\section{A R T I C L E I N F O}

\section{Article history:}

Received 1 March 2017

Received in revised form

10 August 2017

Accepted 19 August 2017

Available online 23 August 2017

\section{Keywords:}

Glutenin

Gliadin

LAOS

Non-linear rheological behavior

\begin{abstract}
A B S T R A C T
Crude gliadin and glutenin fractions were studied using Large Amplitude Oscillatory measurements. LAOS measurements were carried out at three different frequencies $(20,10,1 \mathrm{rad} / \mathrm{sec})$ between the strain values of $0.01-200 \%$. The beginning of non-linearity for glutenin occurred at $\sim 2.5 \%$, while an initial region of strain hardening was observed for gliadin (2.5-10\%) at $1 \mathrm{rad} / \mathrm{sec}$ frequency and up to $15 \%$ at the higher frequencies applied. Lissajous curves showed in the elastic analysis of both fractions glutenin was more elastically dominated since Lissajous curves were narrower, while for gliadin the ellipses were much broader suggesting more fluid-like behavior and each ellipse depended on the magnitude of frequency. Decreasing frequency increased the viscous behavior of both glutenin and gliadin in the nonlinear region, but the change in gliadin was much more pronounced. Gliadin molecules only display intramolecular disulfide bonds creating a great deal of mobility whereas for glutenin molecules, which contain both intermolecular and intramolecular disulfide bonds, the strong network structure formed by this molecular arrangement results in very pronounced strain stiffening.
\end{abstract}

(c) 2017 Elsevier Ltd. All rights reserved.

\section{Introduction}

Wheat gluten proteins (gliadin and glutenin) are responsible for the viscoelastic properties of dough, which develop during mixing. They are primarily responsible for dough's ability to retain gas during fermentation (Verbruggen et al., 1998) because they form strong hydrophobic films. During mixing, hydration, results in changes at the molecular level including interaction between gliadin and glutenin and reorientation of glutenin via S-S interchange (Toufeili et al., 2002). The viscoelasticity of gluten network is known to depend on these intermolecular interactions. Under mechanical action, gradual decay of intermolecular bonds (covalent and non-covalent) causes nonlinear rheological behavior above a critical strain value. The onset of non-linearity starts to occur for wheat flour dough at around $0.2 \%$, whereas it is around $3-10 \%$ for gluten where starch which acts as a filler is absent (Dus and Kokini, 1990; Wang and Kokini, 1995a; Phan-Thien et al., 1997; Phan-Thien et al., 2000 Lefebvre et al., 2000; Uthayakumaran et al., 2002). The gluten network and especially the gliadin fraction is known to be held together by secondary bonding interactions that are

\footnotetext{
* Corresponding author.

E-mail address: kokini@illinois.edu (J.L. Kokini).
}

susceptible to breakdown with increasing strain (Dus and Kokini, 1990). The glutenin fraction is much stronger because it is a complex of high molecular weight storage proteins with molecular weights ranging from 40,000 up to millions depending on the extraction procedure that are held together by a large number of intermolecular disulfide bonds. On the other hand, gliadin is known to be the fraction imparting more viscous character to the gluten network and is held together by intramolecular disulfide bonds (Cocero and Kokini, 1991; Verbruggen et al., 1998).

Several authors have studied the linear and non-linear rheological properties of gluten. Stress relaxation experiments were the most commonly used (Rinde, 1970; Wagner, 1976; Bohlin and Carlson, 1981). To gain more in depth understanding fractions of glutenin and gliadin were extracted by the Osborne method by Cocero and Kokini (1991) and Madeka and Kokini (1994) who developed state diagrams for each gluten fractions. They divided the state diagrams into five regions including glassy region, rubbery region, entangled polymer flow region, reaction region, and the softening region. These state diagrams characterized the entangled polymer flow region when the moisture content of the gluten fractions were higher than $40 \%$ at $25^{\circ} \mathrm{C}$. Wang and Kokini (1995a,b) used the Bird-Carreau model to predict shear viscosity and small amplitude oscillatory measurements and then used the Wagner theory to simulate the steady shear properties for gluten with $55 \%$ 
moisture including shear viscosity and primary nor mal stress coefficient. Lefebvre et al. (2003) reported that gluten showed shear thinning behavior and time-dependent viscosity above a certain creep stress value (when the creep stress exceeds the apparent yield stress, $\sigma \geq 150 \mathrm{~Pa}$ ). Non-linear rheological properties of gluten were also studied. Uthayakumaran et al. (2002) studied the nonlinearity in gluten and wheat flour doughs through elongational properties. They observed 5-7 times higher elongational viscosity for gluten dough when compared to wheat flour dough even at low shear rates. Gluten dough showed higher strain hardening behavior than wheat flour dough when mixed at the optimum water absorption level.

$\mathrm{Ng}$ et al. (2011) applied LAOS tests on gluten and investigated the mechanical properties of gluten. They determined that the onset of non-linearity was after the critical strain at around 5\%. They used Lissajous curves for the first time to evaluate the nonlinear character of gluten. Lissajous curves were also used before by Lefebvre (2006) to evaluate the effect of LAOS deformation on wheat flour dough. The Lissajous curves showed elliptical trajectories for gluten network in the study of $\mathrm{Ng}$ et al. (2011). They observed a rotation in the major axis of the stress ellipse which they have explained as a gradual softening in gluten due to the reduction in network connectivity as the polymer chains were highly stretched.

The earlier LAOS rheological studies of gluten (Ng et al., 2011) do not cover the non-linear behavior of the components of gluten gliadin and glutenins and offer no molecular insights between LAOS data and gluten molecular structure. No study has been carried out to date on the fractions of gluten to characterize their LAOS rheological behavior in detail. In this study we fractionated gluten into its main constituents, gliadin and glutenin, and investigated the non-linear rheological properties of these two main gluten fractions separately by evaluating the Lissajous curves and the resulting LAOS parameters proposed by Ewoldt et al. (2008).

\section{Materials and methods}

\subsection{Materials}

Hard red winter wheat flour (14.64\% moisture, 33.5\% wet gluten, $61.5 \%$ water absorption) obtained from Siemer Milling Company (Hopkinsville, KY) was used to extract gliadin and glutenin fractions.

\subsection{Methods}

\subsubsection{Dough preparation}

Dough samples used for extracting the gluten fractions were prepared using a Farinograph (Brabender, Germany) according to the AACC method No 54-21 (AACC, 2000).

\subsubsection{Extraction of the gluten fractions}

For extracting the gluten fractions, we first separated gluten according to AACC method no 38-10 (AACC, 2000) from the dough sample. Then considering Osborne's wheat protein classification in terms of solubility (Osborne, 1907), gluten was washed with 70\% ethanol solution to separate gliadins and to obtain crude glutenin fraction. Ethanol washing was repeated several times and the sample mixture was centrifuged at $14000 \times \mathrm{g}$ for $10 \mathrm{~min}$ after every washing step. The precipitate is the crude glutenin fraction, where the supernatant is gliadin-ethanol mixture. The gliadin-ethanol solution was evaporated for $6 \mathrm{~h}$ at $40^{\circ} \mathrm{C}$ to prevent denaturation and degradation of gliadin. We obtain a concentrated gliadinethanol mixture which can be easily freeze-dried. Concentrated crude gliadin fraction solubilized in $70 \%$ ethanol and the precipitate which consists of crude glutenin were freeze-dried and then ground to obtain a fine powder. The gluten fraction powders were stored at $-20{ }^{\circ} \mathrm{C}$ until they were needed for the rheological measurements.

\subsubsection{Characterization of the fractions}

Fractions were characterized using SDS-Page gel electrophoresis. In order to establish that the extraction was carried out correctly, SDS-Page electrophoresis was conducted on gluten fractions and on gluten itself. Bullseye $180 \mathrm{kDa}$ protein ladder was purchased from Mid Science Inc. and was used for determining the molecular weights of gluten fractions on the electrophoresis gel.

\subsubsection{Preparation of the fractions for rheological measurements}

An equal weight of water was added to both gliadin and glutenin fractions (100\% of water) in order to prepare dough samples for each protein. They were mixed for $2.5 \mathrm{~min}$ in a Brabender Mixograph with a 30-g cup. These hydrated and mixed protein fraction doughs were used for rheological measurements.

\subsubsection{Non-linear rheological properties}

LAOS measurements were carried out using a DHR-3 Rheometer (TA Instruments, USA). Non-linear properties of the crude gluten fractions were measured between the strain values of 0.01 and $200 \%$ where the applied frequencies were; 10,1 , and $0,1 \mathrm{rad} / \mathrm{sec}$ at $25^{\circ} \mathrm{C}$. A $20 \mathrm{~mm}$-hatch parallel plate geometry and a gap of $2 \mathrm{~mm}$ were used. The measurements were carried out in triplicates and their averages were calculated using Fourier transforms and the inverse transform and used for data analysis. All the non-linear data were obtained through the use of TRIOS software provided by TA for LAOS analysis. Lissajous curves and the stress wave plots were plotted using OriginPro 8.6.

\section{Results and discussion}

\subsection{Extraction and SDS-Page gel electrophoresis}

Low molecular weight glutenin bands appeared around 30-75 kDa and high molecular weight glutenin bands appeared around 130-180 kDa (shown in Supplemental material). The glutenin fraction contains alcohol-insoluble polymeric proteins that have molecular weights changing from 50,000 to $>2$ million. However, it is only possible to visualize around 20 subunit bands on SDS-Page gel when mercaptoethanol is used as a reducer in the sample solvent. This fraction is mainly formed by high molecular weight (HMW) and low molecular weight (LMW) subunits linked by intermolecular disulfide bonds (Osborne, 1907; Wieser et al., 2006). LMW subunits have an aminoacid composition close to $\alpha$-, $\beta$-, and $\gamma$-gliadins. However, HMW glutenin subunits have relatively higher molecular weight than gliadin( 44,000$)$. HMW glutenins have molecular weights ranging between 95,000 and 136,000 (Shewry et al., 1986) and also differ from gliadins by their high content of glycine and low content of proline. Due to their high molecular weight distribution, glutenins are related to dough strength/elasticity, baking performance, and loaf volume (Wieser, 2007). Khan and Bushuk (1979) proposed a model for glutenin which separates glutenin into GluI and GluII fractions. GluI is described as a protein complex that is held together by noncovalent forces. This group of glutenin is reported to have a MW of 68,000 or lower. GluII glutenins consist of larger molecules, where polypeptide subunits are bound with intermolecular disulfide bonds, and also polypeptide aggregates, where subunits are held together by strong noncovalent bonds. GluII glutenins cannot enter SDSPage due to their larger size if not reduced (Osborne, 1907). However, upon the reduction of disulfide bonds, the GluII glutenin 
group dissociate into subunits with a MW of 68,000 or higher, consistent with our SDS results.

Gliadin fractions showed molecular weight values of 5-12 kDa, $25 \mathrm{kDa}, 30-40 \mathrm{kDa}$, and 55-180 kDa for alpha, beta, gamma, and omega gliadin subunits respectively. Gluten shows the combination of all the bands appearing separately for glutenin and gliadin. The major portion of gliadins $(\sim 80 \%)$ consists of monomeric proteins with molecular weights in the range of 30,000 to 55,000 (Osborne, 1907; Wieser et al., 2006; Bozkurt et al., 2014). For this reason, the band appearing for gliadin at around $30 \mathrm{kDa}$ is darker showing a much greater concentration of proteins. Biets and Wall (1972), on the other hand, reported that gliadin proteins have the molecular weight of 16,000 to 50,000 . The fractions $\alpha-, \beta$-, and $\gamma$-gliadins (S (sulphur)-rich gliadins) have lower molecular weights compared to $\omega$-gliadins (S-poor gliadins). $\alpha$-, $\beta$-, and $\gamma$-gliadins showed bands on SDS-Page electrophoresis gel around 32,000 to 42,000 . This value increased up to 44,000 to 72,000 for $\omega$-gliadins (Shewry et al., 1986). These findings are consistent with the SDS-Page gel electrophoresis conducted on crude gluten fractions.

In this study, we studied the LAOS behavior of crude gliadin, and crude glutenin fractions in order to gain deeper insights on the rheology of these two sub-fractions and to attempt to link observed LAOS behavior to the structural differences between the two protein subfractions.

\subsection{Linear and non-linear rheological behavior of crude gliadin and glutenin fractions}

The data in the linear viscoelastic region showed that glutenin is a much more elastic fration compared to gliadin. $G^{\prime}$ values were in the range of $2.5 \times 10^{4}$ to $3.2 \times 10^{4}$ Pa with a $1: 1$ moisture content for glutenin at all frequencies studied. For gliadin on the other hand $G^{\prime}$ values ranged from $1.75 \times 10^{2}$ to $2.45 \times 10^{2}$ Pa a two order of magnitude difference between the two fractions. The phase angle which is a measure of relative solid-like behavior is equal to $19.29^{\circ}$ for gliadin and $10.75^{\circ}$ for glutenin at the frequency of $1 \mathrm{rad} / \mathrm{sec}$; whereas $38.65^{\circ}$ for gliadin and $15.11^{\circ}$ for glutenin at a frequency of $20 \mathrm{rad} / \mathrm{sec}$ clearly showing the relative solid-like behavior of glutenin compared to gliadin at all frequencies studied. As discussed above this is due to the intermolecular disulfide bonds which create a strong network for glutenin and intramolecular disulfide bonds which form a weaker network for gliadin.

Strain sweeps showed that the beginning of non-linearity for glutenin occurred at $\sim 2.5 \%$ strain at all frequencies studied. For gliadin on the other hand an overshoot was observed after $2.5 \%$ strains up to $10 \%$ at a frequency of $1 \mathrm{rad} / \mathrm{sec}$ and up to $15 \%$ at the higher frequencies of 10 and $20 \mathrm{rad} / \mathrm{sec}$. (Fig. 1). $G^{\prime}$ and $G^{\prime \prime}$ values for glutenin did not show a crossover up to the highest applied strain (200\%) at the highest frequency applied (20 rad/sec). However, a crossover modulus value was observed for gliadin at around $41 \%$ strain (at $20 \mathrm{rad} / \mathrm{sec}$ frequency) because of structure decay resulting in increased fluidity as strain is increased. Linear viscoelastic parameters clearly show the stronger network and corresponding elastic character of the glutenin fraction.

\subsection{Analysis of the Lissajous-Bowditch curves}

The intracycle non-linearity for gliadin and glutenin was evaluated at $0.01 \%, 0.06 \%, 1.6 \%, 11 \%, 28 \%, 44 \%, 70 \%, 105 \%$, and $200 \%$ strain. Both the viscous and the elastic Lissajous-Bowditch curves were plotted at these selected strains and at the 3 different applied frequencies. These curves enable us to understand the intracycle rheological behavior of gluten fractions as the amplitude of strain increases.

Fig. 2 shows the raw stress - strain curves plotted as Lissajous curves for both gliadin and glutenin at the three frequencies studied. This data will be normalized and further discussed below. The raw Lissajous curves show that the behavior of gliadin and glutenin are dramatically different. First, the magnitude of the stress amplitude is much larger for glutenin compared to gliadin. For example, for $1 \mathrm{rad} / \mathrm{sec}$ the amplitude of stress for gliadin at $200 \%$ strain is $89 \mathrm{~Pa}$, at $70 \%$ it is $57 \mathrm{~Pa}$, at $1.6 \%$ strain it is $1 \mathrm{~Pa}$. The amplitude of stress at the same frequency and strain values for glutenin is $6163 \mathrm{~Pa}, 3247 \mathrm{~Pa}, 82 \mathrm{~Pa}$ respectively. At the largest frequency of $20 \mathrm{rad} / \mathrm{sec}$ for gliadin, the stress amplitude values are $300 \mathrm{~Pa}, 136 \mathrm{~Pa}, 3.2 \mathrm{~Pa}$ respectively and for glutenin they are $9500 \mathrm{~Pa}$, $5200 \mathrm{~Pa}, 140 \mathrm{~Pa}$. This is roughly consistent with the two orders of magnitude increase in glutenin storage moduli observed in the linear region. We attribute this difference to the networking ability of the two proteins. Clearly gliadin offers much less resistance to deformation and glutenin acts more like a viscoelastic solid due to the differences in their disulfide bond distribution. In addition to the amplitude of stress there is a great deal of information in the shape of the Lissajous curves when we observe their elastic and viscous projection. The elastic projection of gliadin at $10 \mathrm{rad} / \mathrm{s}$ has wider elliptic shapes in the non-linear region and showed a clockwise turn as strain increased, indicating an intracycle strain softening behavior and more liquid like viscous behavior. Glutenin shows much narrower ellipses in the non-linear region indicating a much stiffer/solid like elastic structure; however, it also showed strain softening behavior. The ellipses of gliadin in the viscous projection are narrower than glutenin indicating more liquid like behavior consistent with the elastic projections. Glutenin on the other hand shows fatter ellipses in the viscous projection suggesting more solid like/elastic behavior consistent with the conclusions we reached in the elastic projections. However, both of them showed a clockwise turn as strain increases, which suggests intracycle shear thinning behavior (Ewoldt et al., 2008).

\subsubsection{Normalized elastic projection of Lissajous curves}

Elastic Lissajous curves for gliadin and glutenin are plotted as a function of frequency in Fig. 3. The elliptical trajectories observed for the elastic Lissajous curves of gliadin were wider both in the linear and non-linear regions at all frequencies applied when compared to those of glutenin showing narrower ellipses. As the amplitude of strain increased, the Lissajous curves for both fractions started to become wider because of structure breakdown caused by increased energy input, which means that the elastically dominated behavior deteriorated into more viscous behavior. The elastic Lissajous curves at $20 \mathrm{rad} / \mathrm{sec}$ showed that the nonlinear rheological behavior observed for glutenin at $200 \%$ was still much more elastic than the behavior observed for gliadin in the linear region at the lowest strain $(0.01 \%)$. All these data obtained through the elastic Lissajous curves show that glutenin has much more elastically dominated non-linear viscoelastic behavior compared to gliadin and the decay in elasticity with increasing strain and frequency for glutenin is much slower compared to gliadin because the elliptic loops become wider much more slowly. This is clearly due to the intermolecular disulfide bonds in glutenin that create a strong newtwork compared to gliadin where there are no disulfide covalent bonds to form a 3-D network.

As the frequency decreased, the elliptic loops started to get narrower for both fractions in the linear region (represented by the inner loops) as expected because the structure is reversible in the linear region, whereas the opposite was observed in the non-linear region (represented by the outer loops) and the loops became wider showing irreversibility in the structural changes which occurred caused by the stretching and breaking of covalent bonds as well as ionic, hydrophobic and hydrogen bonding interactions. . Both gluten fractions were more elastic in the linear region and 

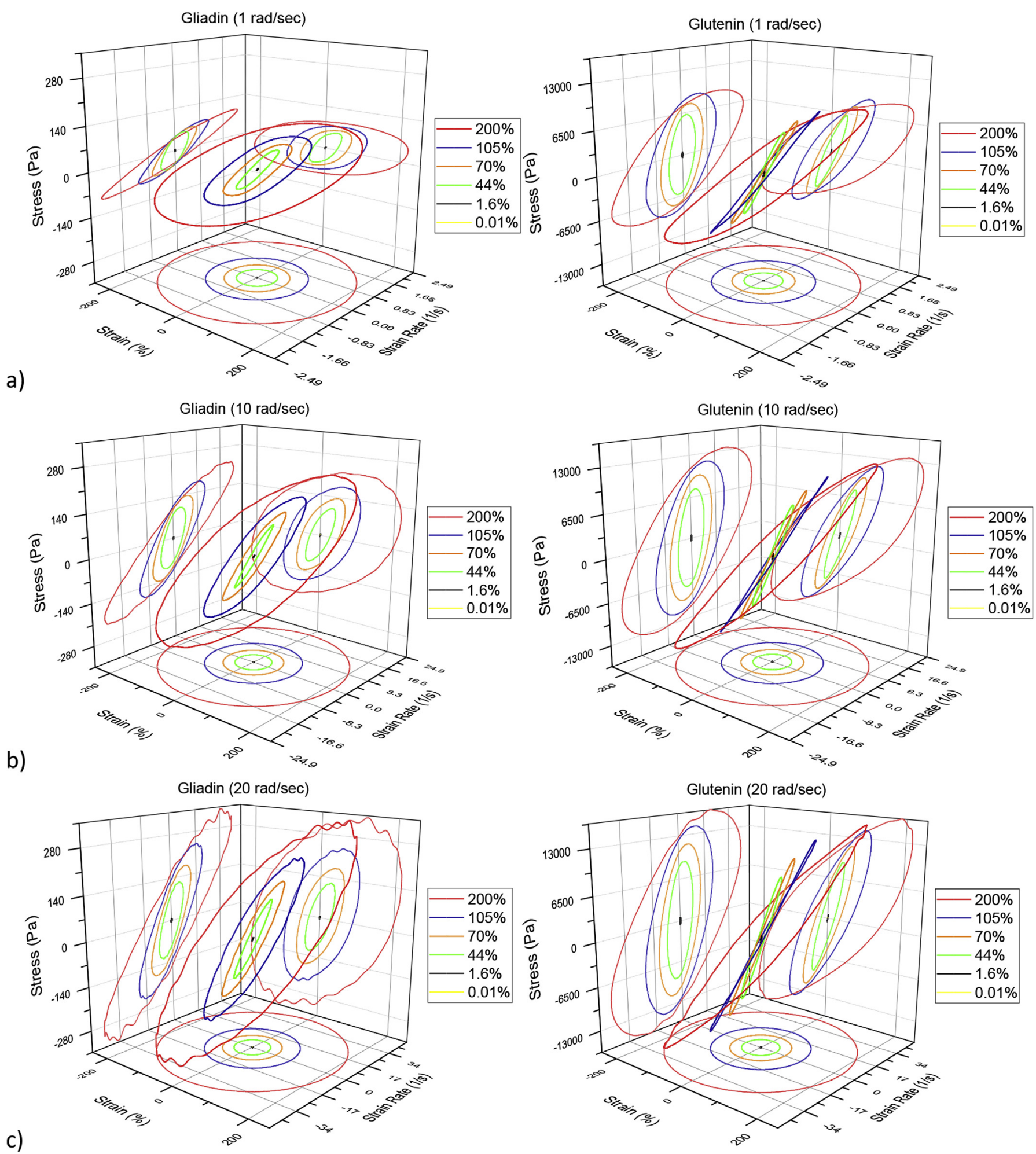

Fig. 2. 3D Lissajous Curves (unnormalized) for gliadin and glutenin at different frequencies; a) $1 \mathrm{rad} / \mathrm{sec}$, b) $10 \mathrm{rad} / \mathrm{sec}$, c) $20 \mathrm{rad} / \mathrm{sec}$.

subunits, especially to HMW subunits, gliadin has fewer constraints to flow and flows readily and consequently is related to the viscous character in dough samples mostly imparting the extensible/ flowable character. On the other hand, having S-rich amino acids and bound to each other by intermolecular bonds, and having higher MW values make glutenin as the responsible fraction for the formation of elastic structure in dough (Shewry et al., 1986; Wieser et al., 2006).

\subsection{Non-linear elastic properties of crude gliadin and glutenin fractions}

$\left(e_{3} / e_{1}\right)$ were $>0(+)$ for both of the gluten fractions, which shows that both gliadin and glutenin showed increasing strain stiffening 

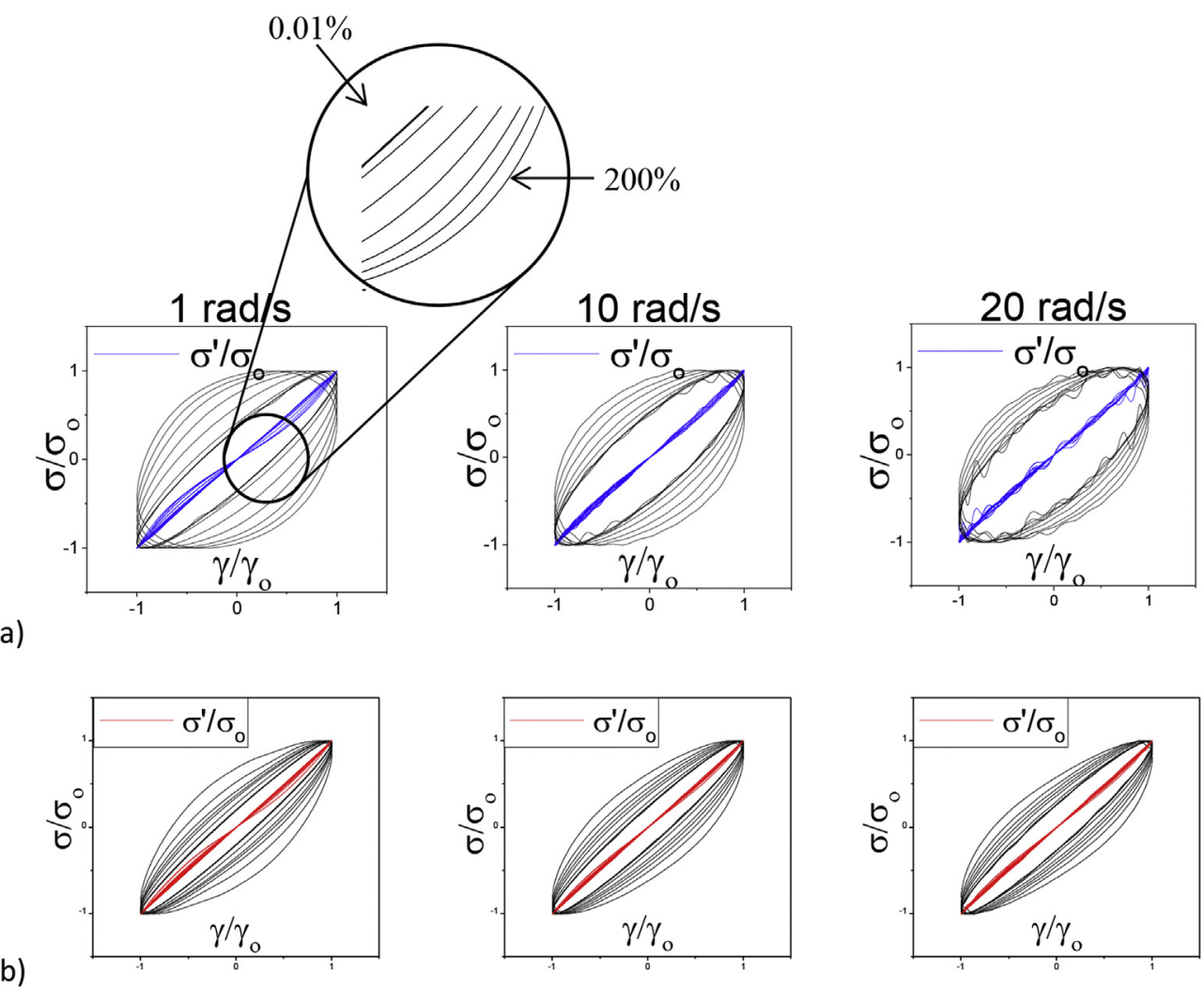

b)
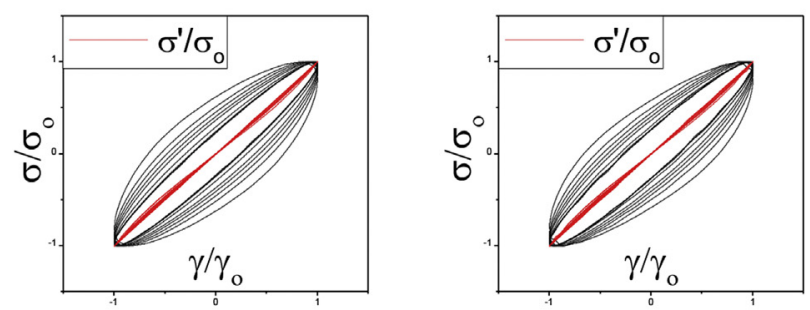

Fig. 3. Lissajous curves for the elastic components of gluten fractions; a) gliadin, b) glutenin.

a)
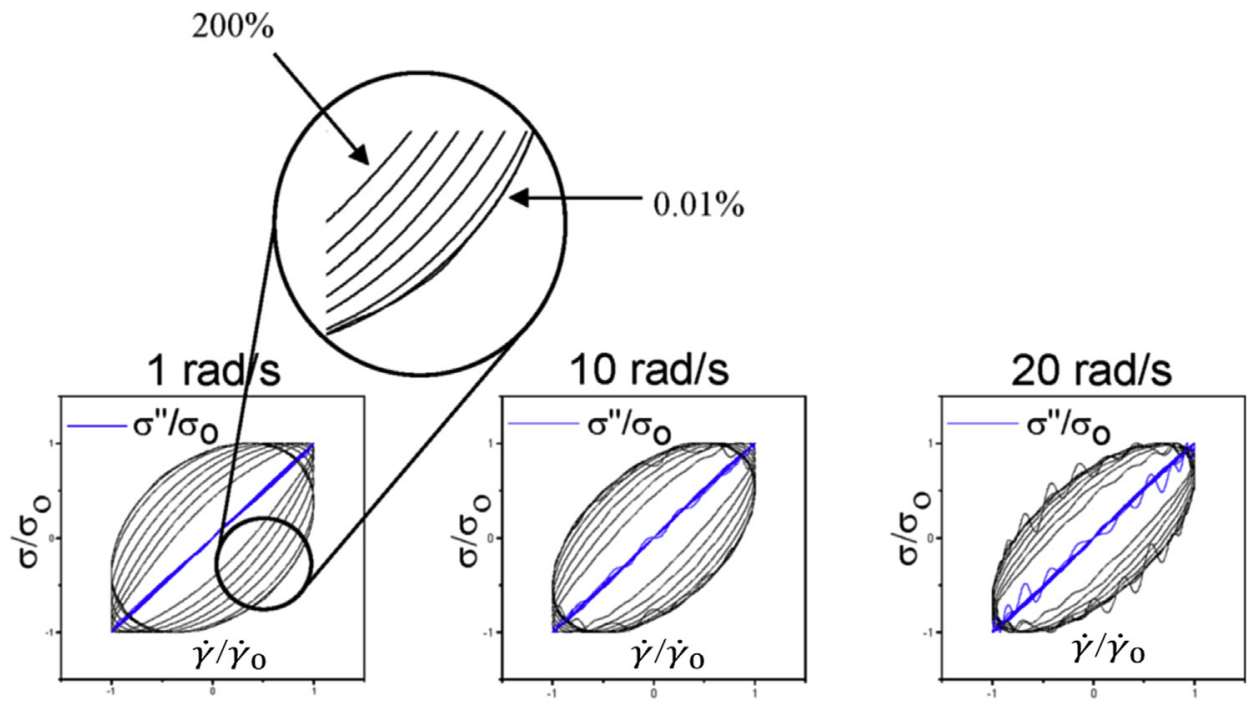

b)
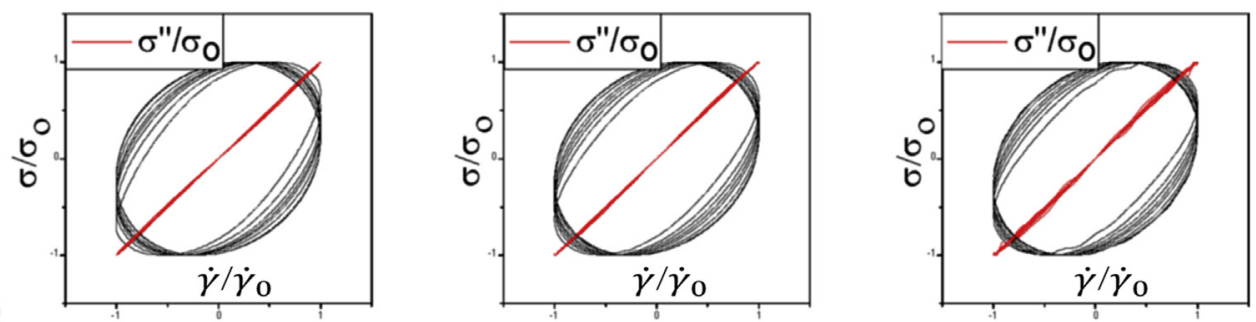

Fig. 4. Lissajous curves for the viscous components of gluten fractions; a) gliadin, b) glutenin. 
behavior in the non-linear region up to peak value. A decrease was observed for gliadin once the strain reached $110 \%$, while the strain stiffening behavior for glutenin never reached a peak value and increased monotonically up to the maximum strain value of $200 \%$ at all frequencies applied (Fig. 5a). A similar strain stiffening behavior was observed for hard wheat flour dough started and a maximum was observed once the strain level reached 70-100\% (Yazar et al., 2016a). These data show that even gliadin fraction shows more elastically dominated non-linear behavior compared to hard wheat flour dough, since the decrease in the strain stiffening behavior starts at $110 \%$ for gliadin. Considering the continuous strain stiffening behavior observed for glutenin, gluten can resist relatively larger strains compared to hard wheat flour dough. This also explains the role of starch in the dough matrix as a filler in the gluten network that softens the structure and explains why the decrease in the strain stiffening behavior occurred at a smaller strain value for dough samples. In an interesting and transformative approach, Phan-Thien et al. (2000) described gluten as a permanent crosslinked network defined by a rubber-like model where rubber-like stress component is combined with a function representing the strain softening behavior which is attributed to starch granules and other loose chains in a dough system. They linked the strong nonlinearity they observed for hard wheat flour dough to its strainsoftening behavior. Dus and Kokini (1990) and Amemiya and Menjivar (1992) attributed non-linearity of dough to the breakdown in the elastic gluten network because of the applied mechanical forces during processing.

The ratio of the $3^{\text {rd }}$ order elastic Chebyshev coefficient to the $1^{\text {st }}$ order elastic Chebyshev coefficients showed a decrease in the strain stiffening behavior of gliadin after the applied strain value of $110 \%$. On the other hand, the permanent cross-linked network definition provided by Phan-Thien et al. (2000) for gluten is considered to be mainly due to the continuously increasing strain stiffening behavior of glutenin we defined by the Chebyshev constants. Large strain modulus $\left(G_{L}^{\prime}\right)$ values were significantly higher for glutenin both in linear and non-linear regions at all frequencies applied (Fig. 5b). For instance, $G_{L}^{\prime}$ values at $1 \mathrm{rad} / \mathrm{sec}$ were $2.10^{1} \pm 0.7 \mathrm{~Pa}$ for gliadin and $3.10^{3} \pm 3.10^{2}$ Pa for glutenin. As the frequency increased, $G_{L}^{\prime}$ values increased for both of the gluten fractions.

\subsection{Non-linear viscous properties of crude gliadin and glutenin fractions}

Gliadin showed shear thinning $\left(v_{3} / v_{1}<0\right)$ behavior in the nonlinear region up to the strain level of $200 \%$ at the frequencies of 10 and $20 \mathrm{rad} / \mathrm{sec}$. At $1 \mathrm{rad} / \mathrm{sec}$, it showed shear thickening behavior between the strain values of $16-113 \%$. Then, it started to show shear thinning behavior again as the strain kept further increasing. Glutenin showed shear thickening $\left(v_{3} / v_{1}>0\right)$ behavior at the lowest frequency applied (1 rad/sec), while shear thinning behavior was observed at higher frequencies (Fig. 5a).

Large strain rate viscosity $\left(\eta_{L}^{\prime}\right)$ values for the crude gluten fractions were plotted against strain rate (Fig. 5c). Similar to $G_{L}^{\prime}$ values, $\eta_{L}^{\prime}$ values for glutenin were significantly higher compared to those of gliadin. However, the effect of the frequency is more pronounced on $\eta^{\prime}{ }_{L}$ values. $\eta_{L}^{\prime}$ values were about the same at $20 \mathrm{rad} / \mathrm{sec}$ and $10 \mathrm{rad} / \mathrm{sec}$ frequency values, while a sharp increase was observed at the lowest frequency applied (1 rad/sec) for both of the gluten fractions.

An approximate $\eta_{L}^{\prime}$ value of $4.10^{2}$ Pa.s was obtained at $1 \mathrm{rad} / \mathrm{sec}$ frequency for the hard wheat flour dough in the previous study (Yazar et al., 2016a), while $\eta_{L}^{\prime}$ value was $2.10^{3} \pm 7.10^{2}$ Pa.s for glutenin and $4.10^{1} \pm 3.5$ Pa.s for gliadin at the same frequency. As observed for the other LAOS parameters, $\eta_{L}^{\prime}$ values also emphasize the highly elastic non-linear rheological behavior in glutenin.
Fig. 6 shows the emergence of third harmonics and the changes occurring in material behavior in the limiting intrinsic region of the LAOS test at the onset of non-linear behavior. We selected the strain range of $0.6 \%-10 \%$ as the intrinsic region for the gluten fractions and scaled the first harmonic moduli $\left(G^{\prime}\right.$ and $\left.G^{\prime \prime}\right)$ using the value of the complex modulus $G^{*}$ obtained in the intrinsic region. Thirdharmonic Chebyshev coefficients $\left(e_{3}\right.$ and $\left.v_{3}\right)$ are scaled by the corresponding linear viscoelastic material function $\left(G^{\prime}=e_{1}\right.$ and $\left.\eta^{\prime}=v_{1}\right)$ at the same frequency within the intrinsic region. Intrinsic LAOS material functions are reported by Ewoldt and Bharadwaj (2013) to be important. They can be related to microstructural differences and physical interpretation of harmonics are best applicable in this region because experimental errors observed at large deformations associated with the magnitude of the third harmonic are minimized since intrinsic regime consists of relatively low strains covering a range from linear to the onset of nonlinearity. They used a single-mode Giesekus model to define the material functions in the intrinsic regime and came up with slope of 2 when $\log \left(e_{3} / e_{1}\right)$ and $\log \left(v_{3} / v_{1}\right)$ are plotted against $\log (\gamma)$. When we plotted $e_{3} / e_{1}$ and $v_{3} / v_{1}$ values for gliadin and glutenin in the intrinsic region, we obtained slope values ranging between 0.45 and 2. Relatively higher slope values were obtained for gliadin and lower values were observed for glutenin.

\section{Conclusion}

LAOS tests were applied to crude gluten fractions extracted using the Osborne classification. These in depth, new and insightful analyses provided a clearer understanding of the functions of gliadin and glutenin in dough rheology arising from the differences in their MWs and amino acid distributions. The elastic and viscous Lissajous curves showed that the changes occurring in the loops were more pronounced for gliadin when compared to glutenin fraction as the samples were imposed to increasing strain and changing frequencies. Both elastic and viscous Lissajous curves for glutenin did not really change against increasing strain and frequencies. That clearly provided an explanation for the flexible fluid structure of gliadin and the stiff character of glutenin.

Elastic Lissajous curves showed that gliadin started to behave more elastically at the lowest strain applied (in the linear region, $0.01 \%$ strain) as the frequency decreased from 20 to $1 \mathrm{rad} / \mathrm{sec}$. If the combination of the lowest frequency and the lowest strain applied is considered as the resting period of a dough sample, gliadin fraction could be the origin of the recovery characteristic of dough with its increasing elastic behavior as the strain and frequency both start to decrease. On the other hand, at the highest strain applied (non-linear region, 200\% strain), the loops are wider for gliadin at all frequencies indicating more viscously dominated non-linear viscoelastic behavior. This flexible character observed for gliadin and the existence of starch as a filler might be an explanation for the decreasing elastically dominated behavior of dough occurring as a result of the mechanical deformations applied during further mixing.

Both gluten fractions showed strain stiffening behavior in the non-linear region $\left(e_{3} / e_{1}>0\right)$. However, a decrease was observed in the strain stiffening behavior of gliadin once the strain reached $110 \%$, while the strain stiffening behavior was continuous for glutenin up to the strain value of $200 \%$ at all frequencies applied. Values obtained for the elastic and viscous LAOS parameters such as $G_{L}^{\prime}$ and $\eta^{\prime}{ }_{L}$ were higher for glutenin at all strain and frequencies applied, which emphasized the stiff character of glutenin together with the Lissajous-Bowditch curves.

Our observations are novel with respect to gliadin and glutenin and in fact, we probed both proteins' rheology in a more depth approach using LAOS in the non-linear region. The new rheological 

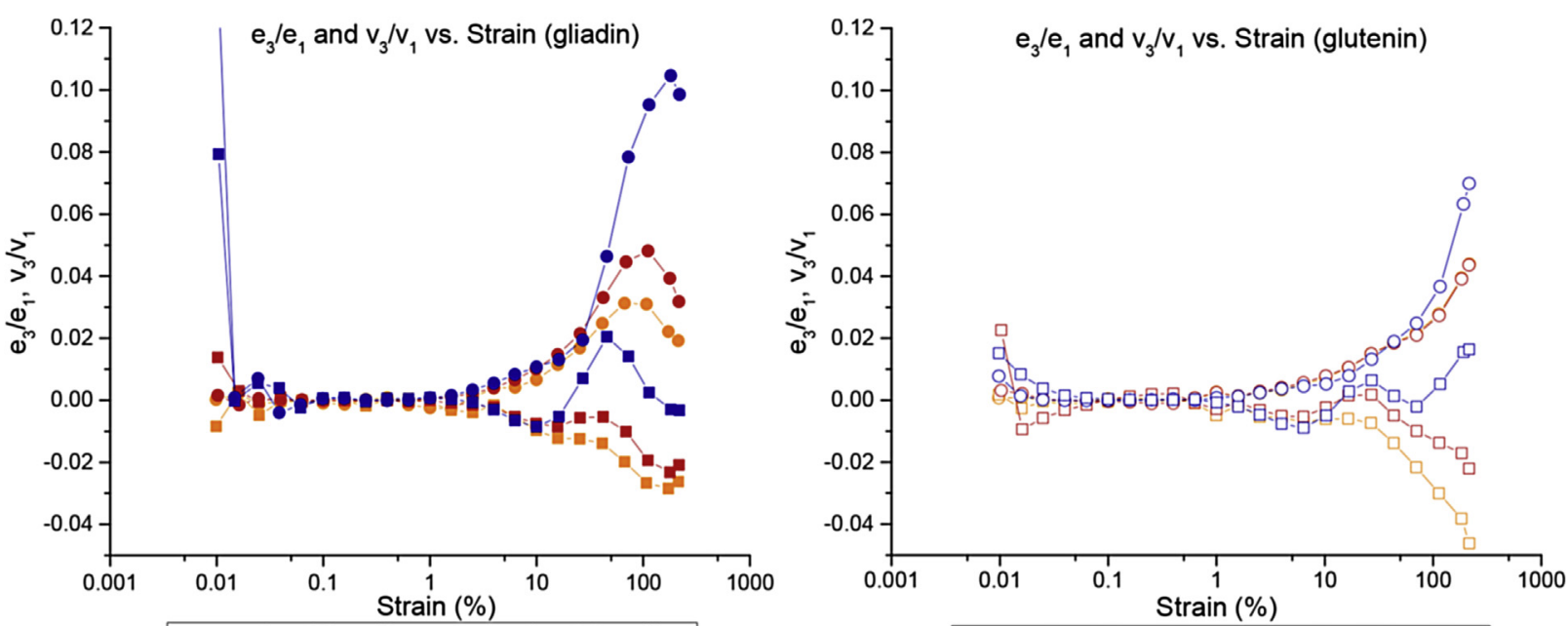

a)
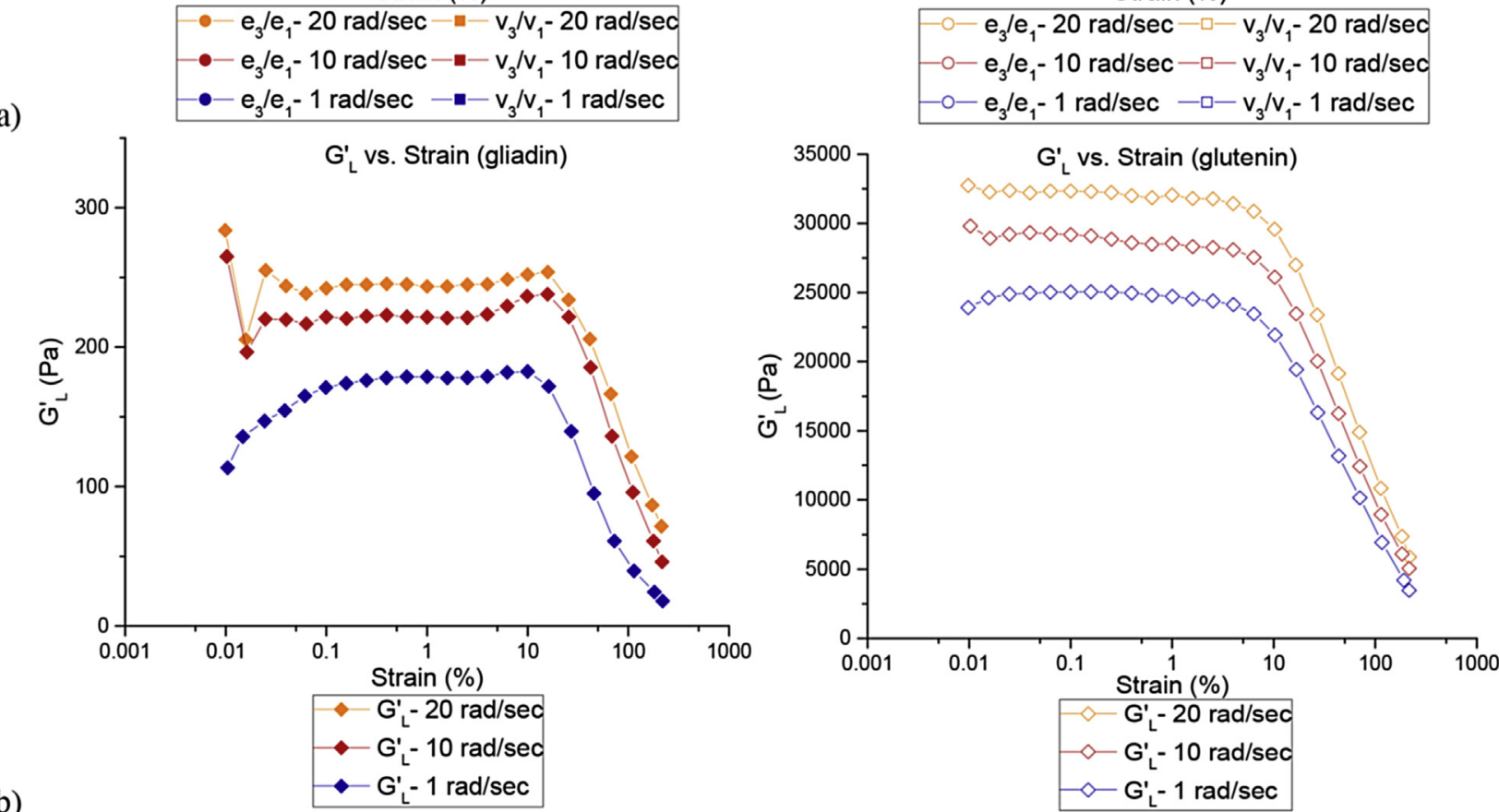

b)
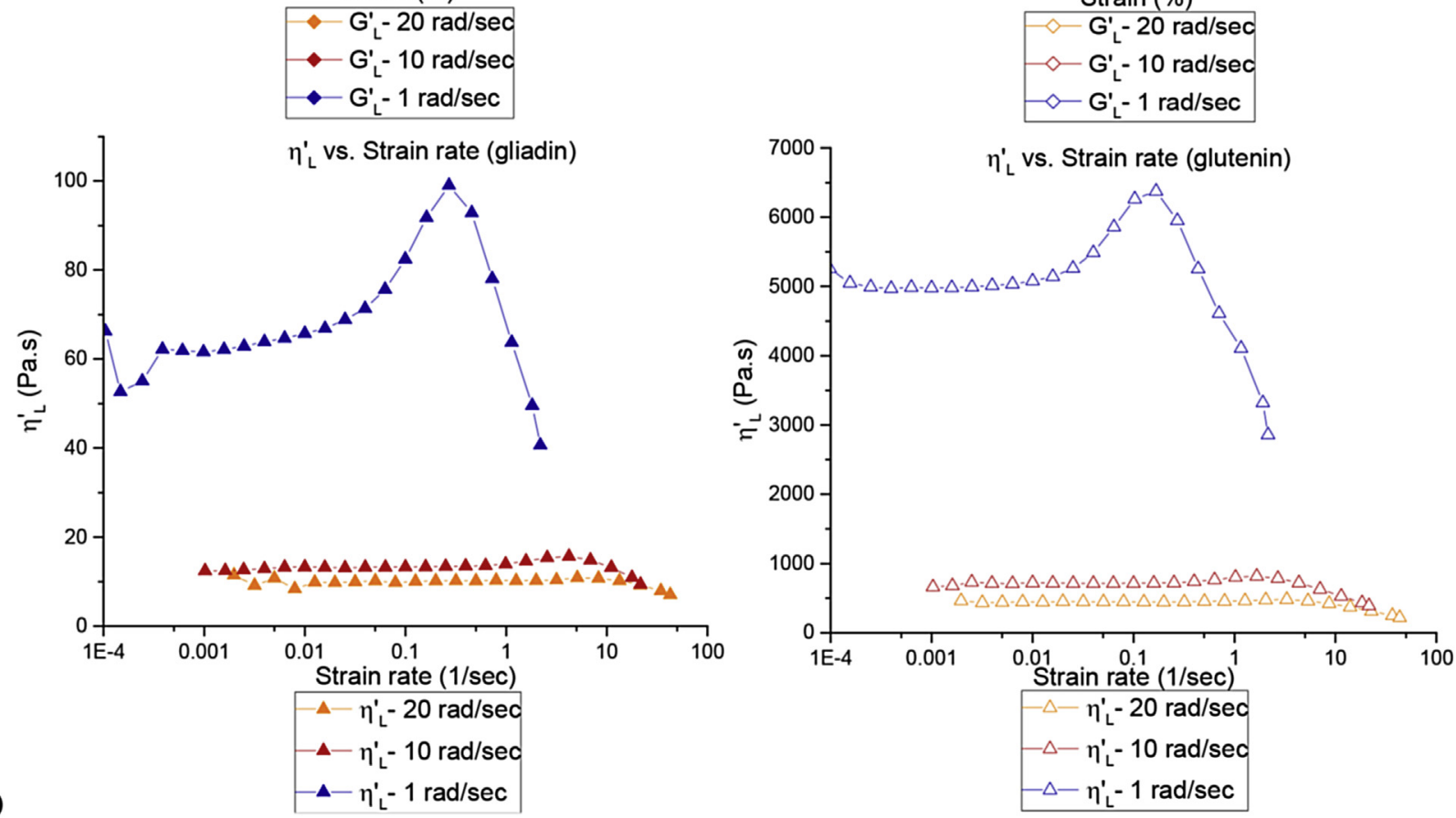

Fig. 5. Elastic and viscous LAOS parameters for the gluten gluten fractions: a) elastic and viscous Chebyshev coefficients, b) $G_{L}^{\prime}$ values, c) $\eta_{L}^{\prime}$ values. 

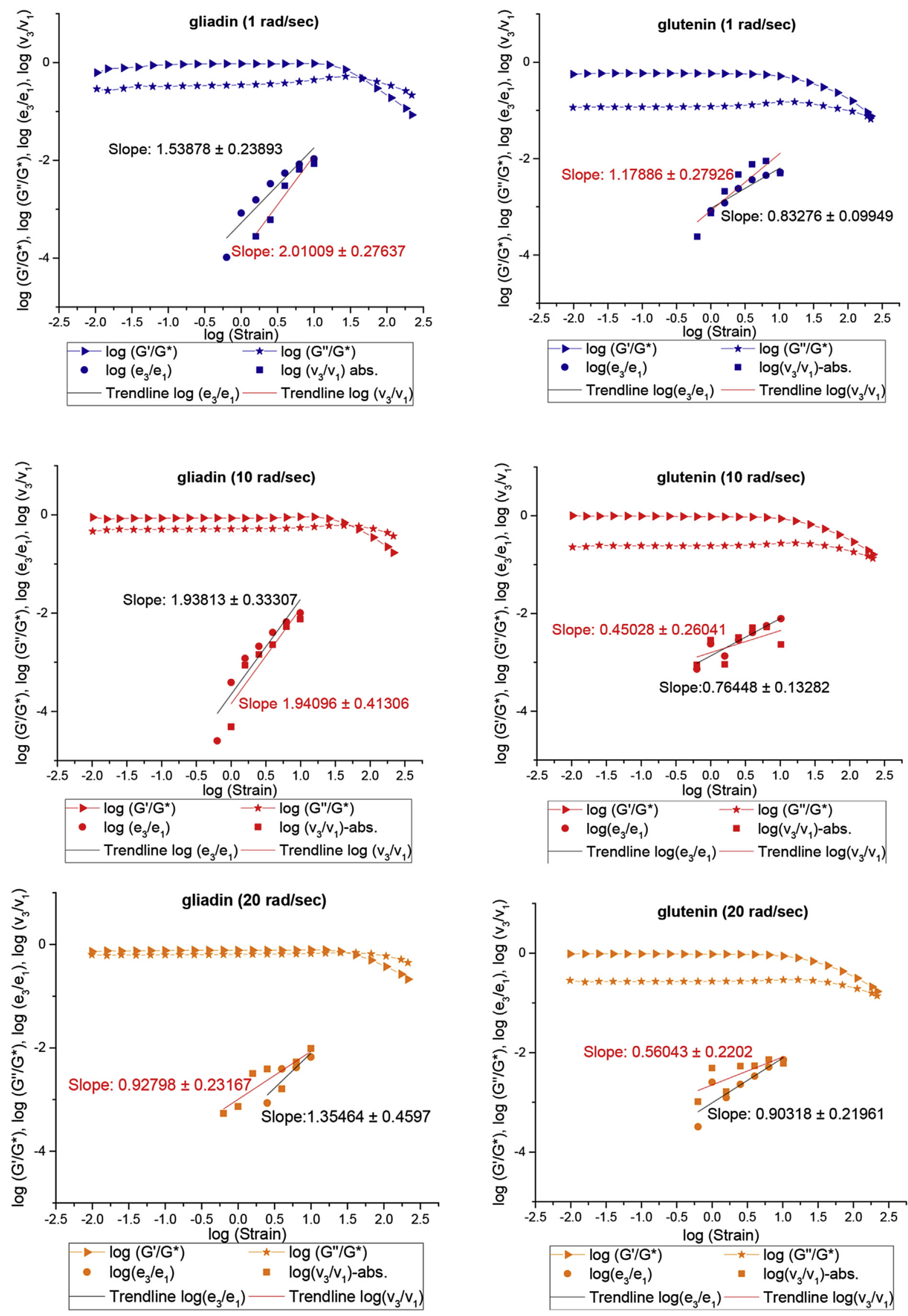

Fig. 6. Elastic and Viscous Chebyshev coefficients for gliadin and glutenin in the intrinsic non-linear LAOS regime. 
insights we gained help understand in more detail the effect of their molecular structure on their flow behavior and probed the effect of intermolecular disulfide bonds resulting in a strong network for glutenin and intramolecular disulfide bonds coupled with secondary bonding interactions (hydrophobic, hydrophilic and ionic) which result in a much weaker network structure for gliadin.

\section{Appendix A. Supplementary data}

Supplementary data related to this article can be found at http:// dx.doi.org/10.1016/j.jcs.2017.08.014.

\section{References}

AACC, 2000. Approved Methods of the AACC, vol. II. American Association of Cereal Chemists, Inc., USA.

Amemiya, J.I., Menjivar, J.A., 1992. Comparison of small and large deformation measurements to characterize the rheology of wheat flour doughs. J. Food Eng. 16, $91-108$.

Biets, J.A., Wall, J.S., 1972. Wheat gluten subunits: molecular weights determined by sodium dodecyl sulfate-polyacrylamide gel electrophoresis. Cereal Chem. 49 (4), 416-430.

Bohlin, L., Carlson, T.L.-G., 1981. Shear stress relaxation of wheat flour dough and gluten. Colloids Surf. 2, 59-69.

Bozkurt, F., Ansari, S., Yau, P., Yazar, G., Ryan, V., Kokini, J.L., 2014. Distribution and location of ethanol soluble proteins (Osborne gliadin) as a function of mixing time in strong wheat flour dough using quantum dots as a labeling tool with confocal laser scanning microscopy. Food Res. Int. 66, 279-288.

Cocero, A.M., Kokini, J.L., 1991. The study of the glass transition of glutenin using small amplitude oscillatory rheological measurements and differential scanning calorimetry. J. Rheol. 25, 257-270.

Dus, S.J., Kokini, J.L., 1990. Prediction of the nonlinear viscoelastic properties of a hard wheat flour dough using the Bird-Carreau constitutive model. J. Rheol. 34 (7), 1069-1084.

Ewoldt, R.H., Hosoi, A.E., McKinley, G.H., 2008. New measures for characterizing nonlinear viscoelasticity in large amplitude oscillatory shear. J. Rheol. 52, $1427-1458$.

Ewoldt, R.H., Bharadwaj, N.A., 2013. Low-dimensional intrinsic material functions for nonlinear viscoelasticity. Rheol. Acta 52, 201-219.

Khan, K., Bushuk, W., 1979. Studies of glutenin. XII. Comparison by sodium dodecyl sulfate-polyacrulamide gel electrophoresis of unreduces and reduced glutenin from various isolation and purification procedures. Cereal Chem. 56 (2), 63-68.

Lefebvre, J., Popineau, Y., Deshayes, G., Lavenant, L., 2000. Temperature-induced changes in the dynamic rheological behaviour and size distribution of polymeric proteins for glutens from wheat near-isogenic lines differing in HMW glutenin subunit composition. Cereal Chem. 77, 193-201.

Lefebvre, J., Pruska-Kedzior, A., Kedzior, Z., Lavenant, L., 2003. A phenomenolical analysis of wheat gluten viscoelastic response in retardation and in dynamic experiments over a large time scale. J. Cereal Sci. 38, 257-267.

Lefebvre, J., 2006. An outline of the non-linear viscoelastic behaviour of wheat flour dough in shear. Rheol. Acta 45 (4), 525-538.

Madeka, H., Kokini, J.L., 1994. Changes in rheological properties of gliadin as a function of temperature and moisture: development of a state diagram. J. Food Eng. 22, 241-252.

Ng, T.S.K., McKinley, G.H., Ewoldt, R.H., 2011. Large amplitude oscillatory shear flow of gluten dough: a model power-law gel. J. Rheol. 55 (3), 627-654.

Osborne, T.B., 1907. The Proteins of the Wheat Kernel (Monograph). Carnegie Inst, Washington, Washington D.C., USA.

Phan-Thien, N., Safari-Ardi, M., Morales-Patino, A., 1997. Oscillatory and simple shear flows of a flour-water dough: a constitutive model. Rheol. Acta 36, 38-48.

Phan-Thien, N., Newberry, M., Tanner, R.I., 2000. Non-linear oscillatory flow of a soft solid-like viscoelastic material. J. Newt. Fluid Mech. 92, 67-80.

Rinde, J.A., 1970. Large-deformation and rupture properties of wheat-flour gluten. Cereal Chem. 47, 225.

Shewry, P.R., Tatham, A.S., Forde, J., Kreis, M., Miflin, B.J., 1986. The classification and nomenclature of wheat gluten proteins: a reassessment. J. Cereal Sci. 4, 97-106.

Toufeili, I., Lambert, I.A., Kokini, J.L., 2002. Effect of glass transition and cross-linking on rheological properties of gluten: development of a preliminary state diagram. Cereal Chem. 79 (1), 138-142.

Uthayakumaran, S., Newberry, M., Phan-Thien, N., Tanner, R., 2002. Small and large strain rheology of wheat gluten. Rheol. Acta 41, 162-172.

Verbruggen, I.M., Veraverbeke, W.S., Vandamme, A., Delcour, J.A., 1998. Simultaneous isolation of wheat high molecular weight and low molecular weight glutenin subunits. J. Cereal Sci. 28, 25-32.

Wagner, M.H., 1976. Analysis of lime-dependent non-linear stress-growth data for shear and elongational flow of a low-density branched polyethylene melt. Rheol. Acta 15, 136-142.

Wang, C.F., Kokini, J.L., 1995a. Prediction of the non-linear viscoelastic properties of gluten doughs. J. Food Eng. 25, 297-309.

Wang, C.F., Kokini, J.L., 1995b. Simulation of the nonlinear rheological properties of gluten dough using the Wagner constitutive model. J. Rheol. 39, 1465-1482.

Wieser, H., Bushuk, W., MacRitchie, F., 2006. The polymeric glutenins. In: Wrigley, C., Bekes, F., Bushuk, W. (Eds.), Gliadin and Glutenin: the Unique Balance of Wheat Quality. AACC International, St. Paul, MN.

Wieser, H., 2007. Chemistry of gluten proteins. Food Microbiol. 24, 115-119.

Yazar, G., Duvarci, O., Tavman, S., Kokini, J.L., 2016a. Effect of mixing on LAOS properties of hard wheat flour dough. J. Food Eng. 190, 195-204.

Yazar, G., Duvarci, O., Tavman, S., Kokini, J.L., 2016b. Non-linear rheological properties of soft wheat flour dough at different stages of farinograph mixing. Appl. Rheol. 26, 1-11. 